\title{
Electric-Field Induced Tricritical Points in Antiferroelectric Liquid Crystal
}

\author{
Andika Fajar* \\ Center for Technology of Nuclear Industry Materials, National Nuclear Energy Agency \\ Kawasan Puspiptek Serpong, Tangerang 15314, Indonesia
}

\begin{abstract}
The electric field effect on the smectic-A - smectic- $\mathrm{C}_{\alpha}$ * phase transition of antiferroelectric liquid crystal have been investigated theoretically. A phenomenological theory under DC field has been developed to establish the calculated hysteresis loop and the $E$ (electric field) $-T$ (temperature)phase diagram, in which a tricritical point was found.
\end{abstract}

KEYWORDS: phase diagram, tricritical point, antiferroelectric liquid crystal, Landau theory

\section{INTRODUCTION}

Since the discovery of the antiferroelectric $\mathrm{SmC}_{A}^{*}$ phase, several new phases have been found in antiferroelectric liquid crystals. Among these, the $\mathrm{SmC}_{\alpha}^{*}$ phase is unique. This phase was already found by DSC measurement in 1989 [1, 2], but it took 10 years to clarify the structure by such sophisticated experiments as resonant X-ray diffraction [3] and differential optical reflectivity [4]. It was found that the $\mathrm{SmC}_{\alpha}^{*}$ phase has a helical structure like the ferroelectric $\mathrm{SmC}^{*}$ phase, but the pitch is very short. For example, it is about three layers in MHPOCBC, which shows the phase sequence as $\mathrm{SmC}_{A}^{*}$ $\mathrm{SmC}_{\alpha}^{*}-\mathrm{SmA}$ as increasing the temperature.

As in solid ferroelectrics, applying an electric field is a good method to investigate the molecular neighboring interactions because in chiral smectic liquid crystals the electric field couples with the order parameter representing the amplitude and phase of the molecular tilt in each layer. Recently a precise $E$ (electric field) $-T$ (temperature) phase diagram of an antiferroelectric liquid crystal MHPOCBC was obtained by means of dielectric measurements [5] as well as by using a photoelastic modulator [6]. In the $\mathrm{SmA}(\mathrm{SmC})$ to $\mathrm{SmC}_{\alpha}^{*}$ transition, there exists a tricritical point (TCP) where the dielectric constant begins to jump discontinously. This tricritical point has been investigated in detail by Bourny et al. [7]. In ferroelectric and antiferroelectric liquid crystal phase transitions the TCP is of great interest from a viewpoint of related anomalies in physical properties.

The phenomenological theories have been developed so far to explain the phase sequences and dynamics properties of chiral smectic liquid crystals [8]. When the free energy is expanded in the Landau-type power series in terms of a single transition parameter, the TCP is simply recognized as the point in a phase diagram where the coefficients of the second order and fourth order terms vanish simultaneously. But, in the case where the free energy is written in terms of more than two transition parameters the situation is rather different, and the procedure for finding TCP is a little more complicated.

The purpose of the present paper is to reexamine the previously studied simple Landau theory written in a term of two parameters for second-order SmA-SmC ${ }_{\alpha}^{*}$ phase transition [9], with the emphasis put on analytical derivation of the TCP, hysteresis loop and then present the $E-T$ phase diagrams in a more transparant way.

\section{ORDER PARAMETER}

First, the order parameter in the $j$-th smectic layer will be defined. The structures of the SmA and SmC phases were shown in Fig. 1. In the SmA phase the molecules are perpendicular to the smectic layers, while in the SmC phase they tilt in the same direction. The molecular tilt can be described by a unit vector parallel to the molecules, called a director $\vec{n}_{j}=\left(n_{j x}, n_{j y}, n_{j z}\right)$. Since opposite orientations of the director are the same, it is natural to use a second rank tensor $n_{i} n_{j}$. From the components we can construct an axial vector:

$$
\vec{\xi}_{j}=\left(\xi_{j x}, \xi_{j y}\right)=\left(n_{j y} n_{j z},-n_{j x} n_{j z}\right)
$$

where the $z$ axis is taken along the layer normal. In the $\operatorname{SmA}$ phase $\vec{\xi}_{j}=0$, while in the SmC phase $\vec{\xi}_{j} \neq 0$. Therefore, $\vec{\xi}_{j}$ is the order parameter describing the SmA-SmC phase transition.

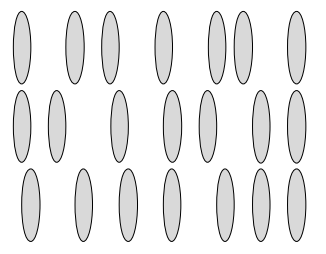

(a) $\mathrm{SmA}$

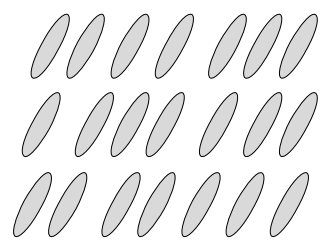

(b) $\mathrm{SmC}$
FIG. 1: Typical smectic phases, (a) SmA and (b) SmC.

${ }^{*}$ E-MAIL: andika@batan.go.id 


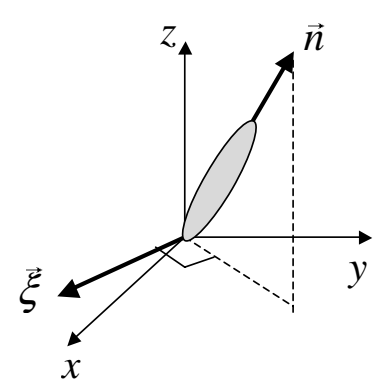

FIG. 2: Relationship between the director $\vec{n}_{j}$ and the order parameter $\vec{\xi}_{j}$.

In chiral smectic liquid crystals, which contain chiral carbons, the tilting direction changes moving along the layer normal and the spontaneous polarization appears in each layer. It is obvious that $\vec{\xi}_{j}$ is always parallel or antiparallel to the polarization. For example, in the ferroelectric phase (SmC*) the molecules tilt as in the SmC phase but $\vec{\xi}_{j}$ rotates slowly to form a helix and the in-plane polarization rotates as well. While in the antiferroelectric phase, the molecules in the neighboring layers tilt in the opposite directions and so the polarizations also point to the opposite directions, and furthermore the chirality causes a small deviation from the $180^{\circ}$ alternation in the tilt between two consecutive layers and the formation of a helical structure as well as in the $\mathrm{SmC}^{*}$ phase.

When an electric field is applied parallel to the smectic layer, two kinds of liquid crystal molecular motion are needed to consider, a spatially homogeneous tilt, i.e., the ferroelectric mode $\left(\xi_{f x}, \xi_{f y}\right)$, and a helicoidal tilt, i.e., the soft mode $\left(\xi_{q 1}, \xi_{q 2}\right)$ related to the $\mathrm{SmA}-\mathrm{SmC}_{\alpha}^{*}$ phase transition, which is the primary order parameter in our case. With these modes the order parameter in the $j$ th layer, $\vec{\xi}_{j}$, can be expressed as [9]

$$
\begin{aligned}
& \xi_{j x}=\xi_{f x}+\xi_{q 1} \cos q_{c} j d-\xi_{q 2} \sin q_{c} j d, \\
& \xi_{j y}=\xi_{f y}+\xi_{q 1} \sin q_{c} j d+\xi_{q 2} \cos q_{c} j d,
\end{aligned}
$$

where $q_{c}$ is the wave number of the helicoidal structure and $d$ the layer spacing. Here, the experimental fact shows that no stripe corresponding to the helical structure was observed with a polarizing microscope in the process of changing the applied field [7]. This indicates that the very short pitch helical structure in the $\mathrm{SmC}_{\alpha}^{*}$ should disappear without the divergence of the pitch, i.e., the amplitude of the helix should become zero. Therefore, $q_{c}$ can be regarded as constant. Similarly, the polarizations corresponding to each relaxation modes are described as,

$$
\begin{aligned}
& P_{j x}=P_{f x}+P_{q 1} \cos q_{c} j d-P_{q 2} \sin q_{c} j d, \\
& P_{j y}=P_{f y}+P_{q 1} \sin q_{c} j d+P_{q 2} \cos q_{c} j d,
\end{aligned}
$$

\section{FREE ENERGY}

The ferroelectric mode located at the Brillouin zone center is directly excited by the applied field through the piezo- electric coupling between the ferroelectric mode and the polarization, which contributes to the linear dielectric response. In the third-order nonlinear dielectric response, on the other hand, the nonlinear coupling between the nonpolar soft mode and the ferroelectric mode plays an essential role. Taking into account these couplings, the free energy $f$ under an applied electric field can be expanded as

$$
\begin{aligned}
f= & \frac{\alpha_{q}^{\prime}}{2} \xi_{q}^{2}+\frac{\beta_{q}}{4} \xi_{q}^{4}+\frac{\alpha_{f}^{\prime}}{2} \xi_{f}^{2}+\frac{\beta_{f}}{4} \xi_{f}^{4}+\frac{\eta}{2} \xi_{q}^{2} \xi_{f}^{2} \\
& -\lambda_{q}\left(\xi_{q 1} P_{q 1}+\xi_{q 2} P_{q 2}\right)-\lambda_{f}\left(\xi_{f x} P_{f x}+\xi_{f y} P_{f y}\right) \\
& +\frac{1}{2 \chi_{q}} P_{q}^{2}+\frac{1}{2 \chi_{f}} P_{f}^{2}-\left(P_{f x} E_{x}+P_{f y} E_{y}\right),
\end{aligned}
$$

where

$$
\begin{array}{r}
\xi_{q}^{2}=\xi_{q 1}^{2}+\xi_{q 2}^{2}, \xi_{f}^{2}=\xi_{f x}^{2}+\xi_{f y}^{2} \\
P_{q}^{2}=P_{q 1}^{2}+P_{q 2}^{2}, P_{f}^{2}=P_{f x}^{2}+P_{f y}^{2}
\end{array}
$$

Here, $\chi_{f}$ the dielectric susceptibility without the coupling between the polarization and ferroelectric order parameter. The $\eta$ term represents the nonlinear biquadratic coupling between the ferroelectric and soft modes. $\lambda_{f}$ is the piezoelectric constant. Equilibrium conditions for the ferroelectric polarization $\left(P_{f x}, P_{f y}\right)$ and $\left(P_{q 1}, P_{q 2}\right)$ yield,

$$
\begin{aligned}
\partial f / \partial P_{f x} & =0 \text { or } P_{f x}=\chi_{f} \lambda_{f} \xi_{f x}+\chi_{f} E_{x} \\
\partial f / \partial P_{f y} & =0 \text { or } P_{f y}=\chi_{f} \lambda_{f} \xi_{f y}+\chi_{f} E_{y} \\
\partial f / \partial P_{q 1} & =0 \text { or } P_{q 1}=\chi_{f} \lambda_{f} \xi_{f x} \\
\partial f / \partial P_{q 2} & =0 \text { or } P_{q 2}=\chi_{f} \lambda_{f} \xi_{f y} .
\end{aligned}
$$

Substituting Eq. (9-12) into Eq. (6), it is obtained

$$
\begin{aligned}
f= & \frac{\alpha_{q}}{2} \xi_{q}^{2}+\frac{\beta_{q}}{4} \xi_{q}^{4}+\frac{\alpha_{f}}{2} \xi_{f}^{2}+\frac{\beta_{f}}{4} \xi_{f}^{4}+\frac{\eta}{2} \xi_{q}^{2} \xi_{f}^{2} \\
& -\chi_{f} \lambda_{f}\left(\xi_{f x} E_{x}+\xi_{f y} E_{y}\right)-\frac{1}{2} \chi_{f}\left(E_{x}^{2}+E_{y}^{2}\right)
\end{aligned}
$$

where $\alpha_{q}=\alpha_{q}^{\prime}-\chi_{q} \lambda_{q}^{2}, \alpha_{f}=\alpha_{f}^{\prime}-\chi_{f} \lambda_{f}^{2}$. When an electric field is applied along the $x$-axis, the free energy density $f$ is given as

$$
\begin{aligned}
f= & \frac{\alpha_{q}}{2} \xi_{q 1}^{2}+\frac{\beta_{q}}{4} \xi_{q 1}^{4}+\frac{\alpha_{f}}{2} \xi_{f x}^{2}+\frac{\beta_{f}}{4} \xi_{f x}^{4}+\frac{\eta}{2} \xi_{q 1}^{2} \xi_{f x}^{2} \\
& -\chi_{f} \lambda_{f} \xi_{f x} E_{x}-\frac{1}{2} \chi_{f} E_{x}^{2}
\end{aligned}
$$

$\xi_{f y}$ has been dropped because it cannot be excited by the field $E_{x}$.

\section{E-T PHASE DIAGRAM}

From the equilibrium conditions, a set of simultaneous nonlinear equations is obtained as

$$
\begin{aligned}
\frac{\partial f}{\partial \xi_{f x}} & =\alpha_{f} \xi_{f x}+\beta_{f} \xi_{f x}^{3}+\eta \xi_{q 1}^{2} \xi_{f x}-\chi_{f} \lambda_{f} E_{x}=0,(15) \\
\frac{\partial f}{\partial \xi_{q 1}} & =\xi_{q 1}\left(\alpha_{q}+\beta_{q} \xi_{q 1}^{2}+\eta \xi_{f x}^{2}\right)=0 .
\end{aligned}
$$


The stability of the equilibrium state obtained is assured if

$$
\begin{aligned}
\frac{\partial^{2} f}{\partial \xi_{f x}^{2}} & =\alpha_{f}+3 \beta_{f} \xi_{f x}^{2}+\eta \xi_{q 1}^{2}>0, \\
\frac{\partial^{2} f}{\partial \xi_{q}^{2}} & =\alpha_{q}+3 \beta_{q} \xi_{q 1}^{2}+\eta \xi_{f x}^{2}>0, \\
\left|H_{i j}\right| & =\left|\begin{array}{cc}
\frac{\partial^{2} f}{\partial \xi_{f x}^{2}} & \frac{\partial^{2} f}{\partial \xi_{f x} \partial \xi_{q}} \\
\frac{\partial^{2} f}{\partial \xi_{f x} \partial \xi_{q}} & \frac{\partial^{2} f}{\partial \xi_{q 1}^{2}}
\end{array}\right| \\
& =\frac{\partial^{2} f}{\partial \xi_{f x}^{2}} \frac{\partial^{2} f}{\partial \xi_{q 1}^{2}}-\left(\frac{\partial^{2} f}{\partial \xi_{f x} \partial \xi_{q 1}}\right)^{2}>0
\end{aligned}
$$

where

$$
\frac{\partial^{2} f}{\partial \xi_{f x} \partial \xi_{q 1}}=2 \eta \xi_{q 1} \xi_{f x} .
$$

and the values representing the concerned equilibrium state have to be substituted for $\xi_{f x}$ dan $\xi_{q 1}$ in $\left|H_{i j}\right|$. The condition for the limit stability of phases, in another words, the phase transition takes place when

$$
\begin{aligned}
\left|H_{i j}\right|= & \left(\alpha_{f}+3 \beta_{f} \xi_{f x}^{2}+\eta \xi_{q 1}^{2}\right)\left(\alpha_{q}+3 \beta_{q} \xi_{q 1}^{2}+\eta \xi_{f x}^{2}\right) \\
& -4 \eta^{2} \xi_{q 1}^{2} \xi_{f x}^{2}=0 .
\end{aligned}
$$

When electric field is applied, $\xi_{f x}$ appears. Therefore under the effect of field SmA phase $\left(\xi_{q 1}=0, \xi_{f x}=0\right)$ has to transform to SmC phase $\left(\xi_{q 1}=0, \xi_{f x} \neq 0\right)$. For large electric field the $\mathrm{SmC}_{\alpha}^{*}$ phase $\left(\xi_{q 1} \neq 0, \xi_{f x} \neq 0\right.$; note that $\xi_{f x}$ is not zero under an external field) becomes less stable, because when $\xi_{f x}$ is large, a nonzero $\xi_{q 1}$ would increase the free energy through $\frac{\eta}{2} \xi_{q 1}^{2} \xi_{f x}^{2}$ term in Eq. (14). This means that only the $\mathrm{SmC}$ phase is stable for large $E$. Therefore there must be exist a phase boundary between the $\mathrm{SmC}_{\alpha}^{*}$ phase and $\mathrm{SmC}$ phase. If $\xi_{q 1}$ vanishes continuously on the phase boundary, the transition is of the second order and the condition for it is expressed, using Eq. (21), as

$$
\frac{\partial^{2} f}{\partial \xi_{f x}^{2}}=\alpha_{f}+3 \beta_{f} \xi_{f x}^{2}=0 \rightarrow \xi_{f x}^{2}=-\frac{\alpha_{f}}{3 \beta_{f}},
$$

or

$$
\frac{\partial^{2} f}{\partial \xi_{q 1}^{2}}=\alpha_{q}+\eta \xi_{f x}^{2}=0 \rightarrow \xi_{f x}^{2}=-\frac{\alpha_{q}}{\eta},
$$

since $\partial^{2} f / \partial \xi_{f x} \partial \xi_{q 1}=0$ for $\xi_{q 1}=0$, where $\xi_{f x}$ is obtained from Eq. (15) with $\xi_{q 1}=0$ by

$$
E_{x}=\frac{1}{\chi_{f} \lambda_{f}}\left(\alpha_{f} \xi_{f x}+\beta_{f} \xi_{f x}^{3}\right),
$$

If $\partial^{2} f / \partial \xi_{f x}^{2}>\partial^{2} f / \partial \xi_{q 1}^{2}$,

$$
\alpha_{f}-\alpha_{q}+\left(3 \beta_{f}-\eta\right) \xi_{f x}^{2}>0,
$$

the phase boundary is determined by Eq. (23) and Eq. (24) which yields,

$$
E_{x}=\frac{1}{\chi_{f} \lambda_{f}} \sqrt{-\frac{\alpha_{q}}{\eta}}\left(\alpha_{f}-\frac{\alpha_{q} \beta_{f}}{\eta}\right)
$$

while if otherwise, by Eq. (22) and Eq. (24).

These apply only to the second order transition. With decreasing temperature, the first order transition may take place from $\mathrm{SmC}_{\alpha}^{*}$ phase to $\mathrm{SmC}$ phase when $E_{x} \neq 0$ even if both $\partial^{2} f / \partial \xi_{f x}^{2}$ and $\partial^{2} f / \partial \xi_{q 1}^{2}$ are still positive. We have to resort to numerical calculations, to some extent, to determine the $\mathrm{SmC}_{\alpha}^{*}$-SmC phase boundary of the first order transition as following.

First the limit stability is determined for the SmC phase when decreasing and $\mathrm{SmC}_{\alpha}^{*}$ phase when increasing the dcfield, respectively. For the SmC phase $\left(\xi_{q 1}=0, \xi_{f x} \neq 0\right)$, Eq. (15) becomes

$$
E_{x}=\frac{1}{\chi_{f} \lambda_{f}}\left(\alpha_{f} \xi_{f x}+\beta_{f} \xi_{f x}^{3}\right) . \text { for } \mathrm{SmC},
$$

where $\xi_{f x}$ is obtained by using Eq. (23) which yield

$$
\xi_{f x 1}^{2}=-\frac{\alpha_{q}}{\eta} .
$$

On the other hand, for the $\mathrm{SmC}_{\alpha}^{*}$ phase $\left(\xi_{q 1} \neq 0, \xi_{f x} \neq 0\right)$, with the use of Eq. (16)

$$
\alpha_{q}+\beta_{q} \xi_{q 1}^{2}+\eta \xi_{f x}^{2}=0 \rightarrow \xi_{q 1}^{2}=\frac{1}{\beta_{q}}\left(-\alpha_{q}-\eta \xi_{f x}^{2}\right)
$$

Eq. (15) becomes,

$$
\begin{aligned}
& E_{a x}=\frac{1}{\chi_{f} \lambda_{f}}\left\{\left(\alpha_{f}-\frac{\alpha_{q} \eta}{\beta_{q}}\right) \xi_{f x}+\left(\beta_{f}-\frac{\eta^{2}}{\beta_{q}}\right) \xi_{f x}^{3}\right\} \\
& \text { for } \mathrm{SmC}_{\alpha}^{*}
\end{aligned}
$$

where the subscript $a$ to $E_{x}$ is used to indicate that it is the field in the $\mathrm{SmC}_{\alpha}^{*}$ phase. $\xi_{f x}$ is obtained by using Eqs. (29),(21) which yield

$$
\xi_{f x 2}^{2}=-\frac{\alpha_{f} \beta_{q}-\alpha_{q} \eta}{3\left(\beta_{f} \beta_{q}-\eta^{2}\right)} .
$$

\section{TRICRITICAL POINT}

Let us consider the TCP related to the transition from $\mathrm{SmC}_{\alpha}^{*}$ phase to $\mathrm{SmC}$ phase, which takes place with decreasing or increasing electric field, is considered. Needles to say, the tricritical point is located on the second order transition line where Eq. (15), Eq. (16) and Eq. (21) are satisfied. Here a general way to determine the TCP is considered. Note that the value of $\xi_{f x}$ in the $\mathrm{SmC}_{\alpha}^{*}$ phase is determined by Eq. (15) (but not by Eq. (16) which is satisfied by any $\xi_{f x}$ if $\xi_{q 1}=0$ ), which is an even function of $\xi_{q 1}$, that is, $d \xi_{f x} / d \xi_{q 1}=0$ at $\xi_{q 1}=0$, the free energy function $f\left(\xi_{f x}, \xi_{q 1}\right)$ can be regarded as a function of a single parameter $\xi_{q 1}$. Then the free energy fucntion can be expanded around $\xi_{q 1}=0$ as

$$
\begin{aligned}
f\left(\xi_{q 1}\right)= & f(0)+\frac{1}{2}\left(\frac{d^{2} f}{d \xi_{q 1}^{2}}\right)_{\xi_{q 1}=0} \xi_{q 1}^{2} \\
& +\frac{1}{4 !}\left(\frac{d^{4} f}{d \xi_{q 1}^{4}}\right)_{\xi_{q 1}=0} \xi_{q 1}^{4}+\cdots
\end{aligned}
$$


since $f$ is an even function of $\xi_{q 1}$.

At the tricrical point, the relation

$$
\begin{aligned}
\left.\frac{d^{2} f}{d \xi_{q 1}^{2}}\right\}_{\xi_{q 1}=0} & =\frac{\partial^{2} f}{\partial \xi_{q 1}^{2}}+\frac{\partial f}{\partial \xi_{f x}}\left(\frac{d^{2} \xi_{f x}}{d \xi_{q 1}^{2}}\right)=0 \\
\left.\frac{d^{4} f}{d \xi_{q 1}^{4}}\right\}_{\xi_{q 1}=0} & =\frac{\partial^{4} f}{\partial \xi_{q 1}^{4}}+6 \frac{\partial^{3} f}{\partial \xi_{f x} \partial \xi_{q 1}^{2}}\left(\frac{d^{2} \xi_{f x}}{d \xi_{q 1}^{2}}\right) \\
& +3 \frac{\partial^{2} f}{\partial \xi_{f x}^{2}}\left(\frac{d^{2} \xi_{f x}}{d \xi_{q 1}^{2}}\right)^{2}=0
\end{aligned}
$$

has to be satisfied, and both relations vanish simultaneously [10]. On differentiating Eq. (15) with respect to $\xi_{q 1}$;

$$
\left.\frac{d^{2}}{d \xi_{q 1}^{2}}\left(\frac{\partial f}{\partial \xi_{q 1}}\right)\right\}_{\xi_{q 1}=0}=\frac{\partial^{2} f}{\partial \xi_{f x}^{2}} \frac{d^{2} \xi_{f x}}{d \xi_{q 1}^{2}}+\frac{\partial^{3} f}{\partial \xi_{f x} \partial \xi_{q 1}^{2}}=0
$$

we obtain

$$
\left.\frac{d^{2} \xi_{f x}}{d \xi_{q 1}^{2}}\right\}_{\xi_{q 1}=0}=-\frac{\frac{\partial^{3} f}{\partial \xi_{f x} \partial \xi_{q 1}^{2}}}{\frac{\partial^{2} f}{\partial \xi_{f x}^{2}}}
$$

and on putting this into Eq. (34), we find the condition of appearance of the TCP as

$$
\left.\frac{d^{4} f}{d \xi_{q 1}^{4}}\right\}_{\xi_{q 1}=0}=\frac{\partial^{4} f}{\partial \xi_{q 1}^{4}}+3 \frac{\partial^{3} f}{\partial \xi_{f x} \partial \xi_{q 1}^{2}} \frac{d^{2} \xi_{f x}}{d \xi_{q 1}^{2}}=0 .
$$

By assuming suitable temperature dependencies of $\alpha_{f}$ and $\alpha_{q}$ such as $\alpha_{q}=A\left(T-T_{c}\right)$ and $\alpha_{f}=\alpha_{q}+b$, where $T$, $T_{c}, A$ and $b$ are the temperature, the transition temperature from the paraelectric SmA phase to the $\mathrm{SmC}_{\alpha}^{*}$ and positive constants, respectively, and using the above equations we can get $\alpha_{f(T C P)}$ as following,

$$
\alpha_{f(T C P)}=\frac{\left(2 \eta^{2}-3 \beta_{f} \beta_{q}\right) b}{2 \eta^{2}-3 \beta_{f} \beta_{q} b+\beta_{q} \eta} .
$$

The $E_{x(T C P)}$ can be obtained by substitung Eq. (38) into Eq. (26). No hysteresis appears for $\alpha_{f(T C P)}<T<T_{C}$ but it does for $T<\alpha_{f(T C P)}$, as shown below.

\section{DISCUSSIONS}

A typical E-T phase diagram made on the basis of the arguments described above is shown in Fig. 3. The adopted values of parameters are given in the caption. The second and first order lines are indicated by solid and dotted lines, respectively. The SmA-SmC $\alpha_{\alpha}^{*}$ second order phase transition takes place at $\alpha_{f}=1$ when $E_{x}=0$. For large electric field, the SmC phase becomes most stable and then $\mathrm{SmC}_{\alpha}^{*}$-SmC phase boundary appears. The part of the second order transition is determined by Eq. (26) because the condition Eq. (25) is satisfied in this case. The TCP is calculated by Eq. (38) and Eq. (26) as $\alpha_{f}=-0.7857$ and $E_{x}=0.4629$. Below this temperature

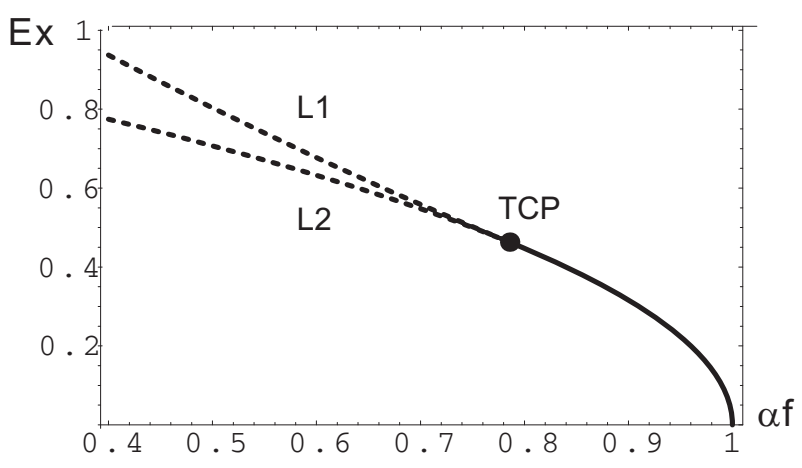

FIG. 3: Theoretically obtained E-T phase diagram, with $\alpha_{f}=\alpha_{q}+$ $1, \beta_{q}=0.3, \beta_{f}=\eta=1, \chi_{f}=1, \lambda_{f}=1$ in the $\mathrm{SmC}_{\alpha}^{*}$ phase .

the first order phase transition from $\mathrm{SmC}_{\alpha}^{*}$ to $\mathrm{SmC}$ has been obtained.

The similar $E-T$ phase diagram has been obtained experimentally by Bourny et al. by the microscopic observation of texture change under dc electric fields [7]. But they cannot draw the line between $\alpha_{f(T C P)}$ (the tempererature of TCP) and $T_{C}$ (the $\mathrm{SmA}-\mathrm{SmC}_{\alpha}^{*}$ phase transition temperature) since no texture change and no stripe were observed when the electric field was gradually increased. It is indicating that in the phase boundary line, the transition from the $\mathrm{SmC}_{\alpha}^{*}$ phase to the unwound SmC phase should take place continuously not through a structure with a large pitch, as usually observed in the second-order phase transition. Below $T_{T C P}$, on the other hand, it is seen the narrow region limited by the lines $L_{1}$ and $L_{2}$ in Fig. 4., which correspondens to the coexisting state of $\mathrm{SmC}_{\alpha}^{*}$ and $\mathrm{SmC}$ phases. Lines $L_{1}$ indicates the phase boundary from $\mathrm{SmC}_{\alpha}^{*}$ to $\mathrm{SmC}$ when increasing the electric field obtained by using Eq. (26). While lines $L_{2}$ is the phase boundary from $\mathrm{SmC}$ to $\mathrm{SmC}_{\alpha}^{*}$ when decreasing the electric field which determined by using Eq. (30) where $\xi_{f x}^{2}$ obtained by using Eq. (29) and Eq. (21). Experimentally it is observed that the ferroelectric SmC domains appeared and then propagated [7]. Therefore, the field-induced transition from $\mathrm{SmC}_{\alpha}^{*}$ phase to $\mathrm{SmC}$ phase is discontinuous with a typical hysteresis.

Next, the double hysteresis loop in the $\mathrm{SmC}_{\alpha}^{*}$ phase will be discussed. The Eqs. (27)-(30) are giving the $\xi_{f x}-E_{x}$ curve together with the stability conditions in Eqs. (18)-(19). The calculated $\xi_{f x}-E_{x}$ curve in the $\mathrm{SmC}_{\alpha}^{*}$ phase depend on the coefficients of the fourth order terms and temperature, and two distinct behavior were observed, as shown in Fig. 4- ??. The $\mathrm{SmC}_{\alpha}^{*}$ and $\mathrm{SmC}$ phases solutions, Eqs. (27)-(30), are stable on the bold lines, and $\xi_{f x 1}$ is given with Eq. (28) and $\xi_{f x 2}$ is given with Eq. (31). In type I $\left(\xi_{f x 2}>\xi_{f x 1}, E_{x}>0\right.$ or $T_{T C P}<T<T_{C}$ ), as shown in Fig. 4 (a)., the field-induced phase transition from $\mathrm{SmC}_{\alpha}^{*}$ phase to $\mathrm{SmC}$ phase, which occurs at $E_{1}^{\prime}$, is of the second order, and therefore, the double hysteresis loop is not observed. In type II ( $\xi_{f x 2}>\xi_{f x 1}, E_{x}>$ 0 or $T<T_{T C P}$ ), as shown in Fig. 4 (b) and (c), on the other hand, the transition is of the first order. Since there exist two critical points, $P_{a}$ and $P_{f}$, at which the solution become unstable, the double hysteresis curve appears. This result show the existence of TCP. 


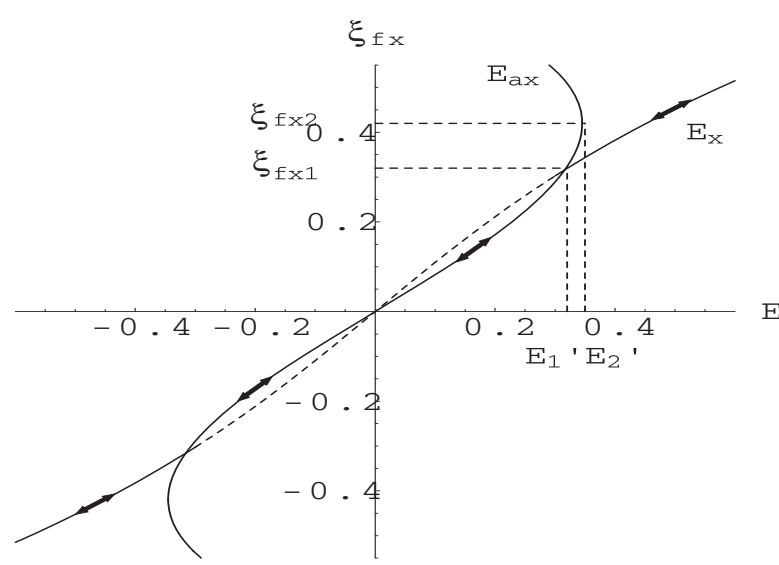

(a)

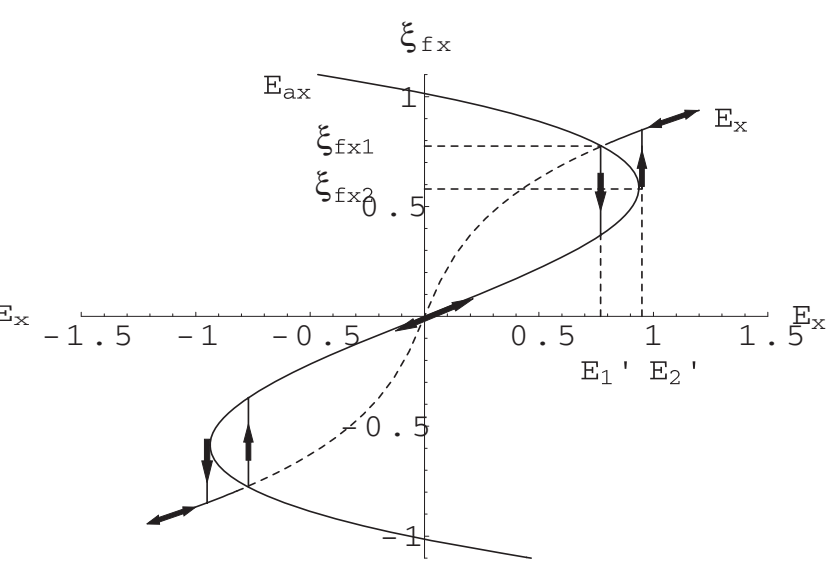

(b)

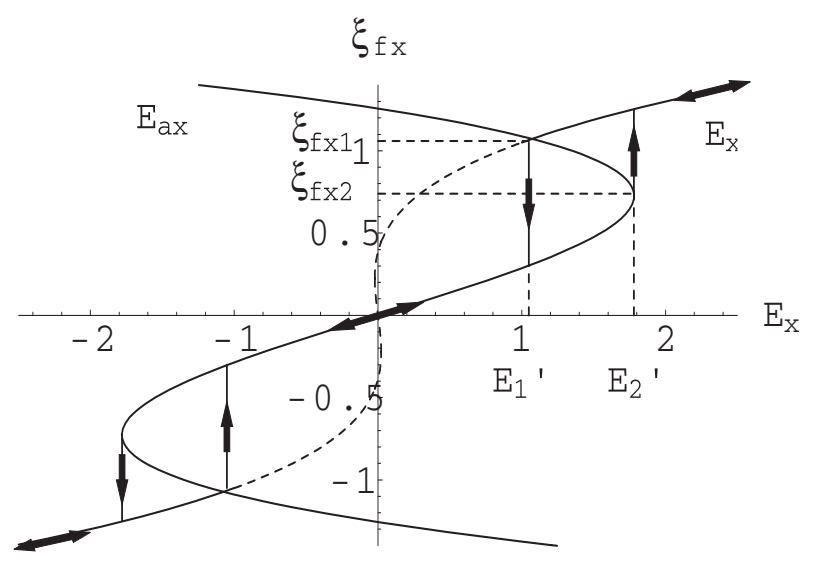

(c)

FIG. 4: Calculated hysteresis loop at (a). $\alpha_{f}=0.9^{\circ} \mathrm{C}$, (b). $\alpha_{f}=0.4^{\circ} \mathrm{C}$, (c). $\alpha_{f}=-0.15^{\circ} \mathrm{C}$, with $\alpha_{f}=\alpha_{q}+1, \beta_{q}=0.3, \beta_{f}=\eta=1, \chi_{f}=1$, $\lambda_{f}=1$ in the $\mathrm{Sm}-C_{\alpha}^{*}$ phase.

\section{CONCLUSION}

The $E-T$ phase diagram of MHPOCBC near the SmA$\mathrm{SmC}_{\alpha}^{*}$ transition point has been established and a tricritical point has been found . No hysteresis appears for the temperature between $\alpha_{f(T C P)}$ and $T_{C}$ but it does for the temperature below $\alpha_{f(T C P)}$.
[1] M. Fukui, H. Orihara, N. Yamamoto, Y. Yamada, and Y. Ishibashi, Jpn. J. Appl. Phys. L28, 849 (1989).

[2] E. Gorecka, A. D. L. Chandani, Y. Ouchi, H. Takezoe, and A. Fukuda, Jpn. J. Appl. Phys. 29, 131 (1990).

[3] P. Mach, R. Pindak, A. M. Levelut, P. Barois, H. T. Nguyen, C. C. Huang, and L. Furenlid, Phys. Rev. Lett. 81, 1015 (1998).

[4] A. Cady, X. F. Han, D. A. Olson, H. Orihara, and C. C. Huang, Phys. Rev. Lett. 91, 125502 (2003).

[5] A. Fajar and H. Orihara, Mol. Cryst. Liq. Cryst. 511, 239 (2009).
[6] H. Orihara, Y. Naruse, M. Yagyu, A. Fajar, and S. Uto, Phys. Rev. E. 72, 040701 (2005).

[7] V. Bourny and H. Orihara, Phys. Rev. E 63, 021703 (2001).

[8] I. Musevic, R. Blinc and B. Zeks, The Physics of Ferroelectric and Antiferroelectric Liquid Crystals, World Scientific Publishing Co. Pte. Ltd. (2000) .

[9] V. Bourny, A. Fajar, and H. Orihara, Phys. Rev. E 62, R5903 (2000).

[10] I. Suzuki and Y. Ishibashi, J. Phys. Soc. Jpn. 52, 2088 (1983). 